\title{
Wetting of a symmetrical binary fluid mixture on a wall
}

\author{
F. Schmid \\ Max-Planck Institut für Polymerforschung, D-55021 Mainz, Germany \\ Fakultät für Physik, Universität Bielefeld, D-33615 Bielefeld, Germany \\ N. B. Wilding \\ Department of Physics and Astronomy, The University of Edinburgh, \\ Edinburgh EH9 3JZ, U.K. \\ Department of Mathematical Sciences, The University of Liverpool, \\ Liverpool L69 7ZL, U.K.
}

\begin{abstract}
We study the wetting behaviour of a symmetrical binary fluid below the demixing temperature at a non-selective attractive wall. Although it demixes in the bulk, a sufficiently thin liquid film remains mixed. On approaching liquid/vapour coexistence, however, the thickness of the liquid film increases and it may demix and then wet the substrate. We show that the wetting properties are determined by an interplay of the two length scales related to the density and the composition fluctuations. The problem is analysed within the framework of a generic two component GinzburgLandau functional (appropriate for systems with short-ranged interactions). This functional is minimized both numerically and analytically within a piecewise parabolic potential approximation. A number of novel surface transitions are found, including first order demixing and prewetting, continuous demixing, a tricritical point connecting the two regimes, or a critical end point beyond which the prewetting line separates a strongly and a weakly demixed film. Our results are supported by detailed Monte Carlo simulations of a symmetrical binary Lennard-Jones fluid at an attractive wall.
\end{abstract}

PACS numbers 68.45.Gd, 68.10.-m, 05.70.Jk, 68.15.+e

\section{INTRODUCTION}

Phase transitions are well known to be influenced by geometrical confinement [1]. In practice, confinement is often imposed by rigid external constraints, for example the surfaces of porous or artificially nanostructured media. However, it can also be an inherent feature of a system, as occurs for a liquid wetting film bound to a solid substrate and in equilibrium with its vapour [2]. In such a situation the liquid is confined between the rigid substrate and the flexible liquid-vapour interface.

The effects of confinement are particularly pronounced in the region of critical points. Under such conditions the system exhibits strong order parameter fluctuations, the correlation length of which may become comparable with the linear dimension of the confined system. When this occurs the effects of confinement are felt not just near the confining surfaces, but propagate throughout the system [3].

Critical fluctuation are relevant to the properties of liquid wetting films if the liquid in question possesses an additional internal degree of freedom. Then the state of the liquid is described not only by its number density, but by an additional parameter measuring the degree of internal order. Examples are binary liquids, where the additional order parameter is the relative concentration of species, and ferrofluids where it is the magnetisation. In such systems the geometrical constraint (i.e. the film thick- ness) can itself depend on the state of order in the liquid film. For example, simulation and experiment have recently shown that critical concentration fluctuations can change the equilibrium thickness of a wetting layer of a binary liquid - the so-called critical Casimir effect $\left[\begin{array}{l}\text { (6) } \\ \text {. }\end{array}\right.$.

It transpires, however, that the interesting consequences of interplay between the degree of order in the wetting layer and film thickness are not limited to the critical point itself. To illustrate this, it is instructive to consider the following Gedanken experiment. Let us take a symmetrical binary fluid, i.e. a fluid in which particles of the same species have one strength of interaction, while interactions between dissimilar species have another strength. As elucidated in ref. [7], it is possible to arrange for such a system to exhibit a line of continuous demixing transitions, terminating in a critical end point on the liquid side of liquid-vapour coexistence. Suppose now that the fluid is placed in contact with a non-selective attractive substrate (wall) acting equally on both species. If the wall is sufficiently attractive, complete wetting occurs at and above the critical end point temperature $T_{c e p}$ as liquid-vapour coexistence is approached from the vapour side. But what happens for $T<T_{c e p}$ ? Far from coexistence, the wetting film is sufficiently thin that demixing will certainly be suppressed. On approaching the coexistence curve, however, the film 
thickness grows and it is tempting to argue that it eventually exceeds the correlation length of composition fluctuations, whereupon the film spontaneously demixes.

Notwithstanding the appealing simplicity of this argument, it turns out to contain two flaws which render the actual situation rather more complex. First, the thickness of the mixed wetting film will not increase beyond all limits below the critical end point $T_{\text {cep }}$. This is because a hypothetical mixed bulk liquid would not coexist with the vapour phase at the same chemical potential $\mu_{0}$ as a demixed liquid, but rather at a chemical potential which is shifted by

$$
\mu_{*}-\mu_{0} \propto\left(T_{c e p}-T\right)^{2-\alpha}
$$

towards the liquid side in the $\mu-T$ plane [8,9]. Since the thickness of the mixed film grows as $\ln \left(\mu_{*}-\mu\right)$ [2, 10], it is bounded from above by

$$
l_{*} \propto \ln \left(\mu_{*}-\mu_{0}\right) \propto \ln \left(T_{c e p}-T\right) .
$$

The maximum thickness $l_{*}$ of a hypothetical mixed film thus diverges logarithmically on approaching $T_{\text {cep }}$. In contrast, the correlation length $\xi$ diverges much faster, like $\xi \propto\left(T_{c e p}-T\right)^{-\nu}$ and will thus always exceed $l_{*}$ sufficiently close to $T_{\text {cep }}$.

The second flaw in our argument is its implicit assumption that the composition or order parameter profile is confined in an effectively steplike density profile, i.e. that the interfacial width between the liquid and the vapour is much smaller than the correlation length of composition fluctuations. Although this is true in the region of the critical end point, for temperature sufficiently below $T_{c e p}$ the correlation lengths of density and composition fluctuations can be comparable and the interplay between the two subtle.

In the present study, we deploy mean field calculation and Monte Carlo simulation to elucidate the range of possible wetting behaviour of a symmetrical binary fluid mixture at a non-selective attractive wall for temperatures below $T_{\text {cep }}$. For the sake of simplicity, we have chosen to ignore long range dispersion forces in the analytical calculations, instead taking the interactions to be short ranged. This allows us to base our study on a generic Ginzburg-Landau model which we solve numerically and analytically within a square gradient approximation. The latter leads to the construction of a film free energy (effective interface potential) highlighting the role of the different length scales involved in the problem. We show that the competition of length scales results in wetting phase behavior considerably more complex than has hitherto been appreciated. The analytical results are compared with (and supported by) detailed Monte Carlo simulations of a binary Lennard-Jones fluid in a semi-infinite geometry, interacting with a non-selective attractive substrate via dispersion forces.

With regard to previous related work, the sole discussion of wetting of symmetrical binary fluids at a nonselective wall (of which we are aware) is that of Dietrich and Schick 11] who considered them in a sharp kink approximation treatment of binary fluids having longranged interactions. Most other work on the wetting properties of binary fluids has focused on the case of a selective substrate (favouring one component) [10 14]. Although such models correspond more closely than ours to experimental conditions 10,15, they lack the aspect of simultaneous demixing/ordering and wetting which is of interest to us here. It should be stressed, however, that realisations of fluids having symmetrical internal degrees of freedom do in fact exist, notably in the form of ferrofluids [16], so our model is of more than purely theoretical interest.

More general studies of wetting in systems with more than one order parameter and associated length scales have been discussed by Hauge [17], who pointed out that wetting exponents may become nonuniversal even on the mean field level due to the competition of length scales. Later studies have often focused on this nonuniversality, e.g. in the context of wetting phenomena in superconductors 18], alloys [19,20 and related systems [21].

The present paper is organised as follows. In section III we introduce our Ginzburg-Landau free energy functional and obtain its wetting behaviour in the limits of infinite and vanishing order parameter stiffness. At intermediate values of the stiffness parameter the wetting behaviour is found firstly via an analytical minimisation of the functional within a piecewise parabolic potential approximation (sec. II B), and then (in sec. II Q) via a numerical minimisation of the free energy functional to obtain the density/order parameter profiles. In section III A we report the results of grand canonical Monte Carlo studies of a symmetrical binary Lennard-Jones fluid at an attractive structureless wall. The density and order parameter profiles with respect to the wall are obtained along a sub-critical isotherm for a number of different wall-fluid potential strengths. Finally we compare and discuss the mean-field and simulation results in section IV.

\section{GINZBURG-LANDAU THEORY}

Our theoretical studies are based on a generic Ginzburg-Landau functional for a system with two order parameters $\phi(\vec{r}, z)$ and $m(\vec{r}, z)$ :

$$
\begin{aligned}
\mathcal{F}=\int d \vec{r} & \int_{0}^{\infty} d z\left\{\frac{g}{2}(\nabla \phi)^{2}+\frac{\gamma}{2}(\nabla m)^{2}+f(m, \phi)\right\} \\
& +\left.\int d \vec{r} f_{s}(m, \phi)\right|_{z=0}
\end{aligned}
$$

with the bulk free energy density

$$
\begin{aligned}
f(m, \phi)= & -\frac{a_{\phi}}{2} \phi^{2}+\frac{b_{\phi}}{4} \phi^{4}-\frac{a_{m}}{2} m^{2}+\frac{b_{m}}{4} m^{4} \\
& +\mu \phi-\kappa m^{2} \phi
\end{aligned}
$$

and the bare surface free energy at the wall 


$$
f_{s}(m, \phi)=\frac{C_{\phi}}{2} \phi^{2}+H_{\phi} \phi+\frac{C_{m}}{2} m^{2} .
$$

The $z$-axis is taken to be perpendicular to the wall and $\int d \vec{r}$ integrates over the remaining spatial dimensions. In our case, the quantity $m$ is related to the difference between the partial densities of the two components, $m \propto\left(\rho_{A}-\rho_{B}\right)$, and $\phi$ to the total density, $\phi \propto\left(\rho-\rho_{0}\right)$, where the reference density $\rho_{0}$ is chosen in the liquid/vapour coexistence region such the cubic term proportional to $\left(\rho-\rho_{0}\right)^{3}$ in (2.2) vanishes. Below the liquid/vapour critical point, it is convenient to set the units of $\phi, m, F$ and of the length such that $b_{m}=b_{\phi}=a_{\phi}=g=1$, and to define $\theta=a_{m}-1$. The bulk free energy density then takes the form

$$
\begin{aligned}
f(m, \phi)= & -\frac{1}{2} \phi^{2}+\frac{1}{4} \phi^{4}-\frac{\theta}{2} m^{2}+\frac{1}{4} m^{4} \\
& -\mu \phi+\kappa(1-\phi) m^{2} .
\end{aligned}
$$

The bulk properties of this model have been discussed earlier [7]. A $\lambda$-line $\theta_{\lambda}(\mu)$ of continuous transitions separates the mixed fluid from the demixed fluid at large negative $\mu$, corresponding to large densities $\phi$. As long as $\kappa<1$, it is terminated by the onset of liquid/vapour coexistence at a critical end point $\left(\theta_{c e p}=0, \mu_{c e p}=0\right)$. The parameter $\mu$ is field like and $\theta$ is temperature like, $\theta \propto\left(T-T_{c e p}\right)$, where $T_{c e p}$ is the critical end point temperature. Above $\theta_{c e p}$, liquid/vapour coexistence is encountered at $\mu=0$, and below $\theta_{c e p}$, at

$$
\mu_{c}=\frac{\theta^{2}}{8\left(1-\kappa^{2}\right)} .
$$

The coexisting liquid and gas phases are characterized by the order parameters (to linear order in $\mu$ )

$$
m_{-}^{*}=0 \quad \phi_{-}^{*}=-1-\mu / 2
$$

in the gas phase, and

$$
m_{+}^{*}=\frac{\theta-\kappa \mu}{1-\kappa^{2}} \quad \phi_{+}^{*}=1-\frac{\mu}{2}+\frac{\kappa}{2} m_{+}^{* 2}
$$

in the liquid phase. These expressions are also valid in the regime where the liquid or gas phase are metastable.

Minimizing the functional (2.1) yields the Euler Lagrange equations

$$
g \frac{d^{2} \phi}{(d z)^{2}}=\frac{\partial f}{\partial \phi} \quad \gamma \frac{d^{2} m}{(d z)^{2}}=\frac{\partial f}{\partial m}
$$

with the boundary conditions

$$
\left.g \frac{d \phi}{d z}\right|_{z=0}=\left.\frac{\partial f_{s}}{\partial \phi} \quad \gamma \frac{d m}{d}\right|_{z=0}=\frac{\partial f_{s}}{\partial m} .
$$

We wish to study a situation where the mixed liquid $(m \equiv 0)$ wets the wall at $\mu=0$ (coexistence between vapour and mixed liquid). To ensure this under all circumstances, we choose $H_{\phi}=-\phi_{0} C_{\phi}$ with $\phi_{0}>\phi_{+}^{*}$ and take the limit $C_{\phi} \rightarrow \infty$, which is equivalent to constraining the surface density at the fixed value $\phi(0)=\phi_{0}$. The surface coupling $C_{m}$ is taken to be positive. It accounts for weakening of the demixing tendency at the surface due to the reduced number of interacting neighbors.

One possible solution of the Euler Lagrange equations describes a mixed film at a wall. In this case, $m(z)=$ 0 everywhere and one is left with one order parameter $\phi$ only. The bulk value of $\phi$ in the metastable mixed phase is $\phi_{+}^{(0)}=1-\mu / 2$. The standard way of solving the problem [2] shall be sketched briefly for future reference. One begins by identifying the integration constant

$$
\frac{1}{2}\left(\frac{d \phi}{d z}\right)^{2}-f(\phi)+f\left(\phi_{-}^{(0)}\right) \equiv 0 .
$$

which gives an expression for $d \phi / d z$ as a function of $\phi$. The surface free energy can then be expressed as an integral over $\phi$

$$
F_{e x c}^{(0)}=\int_{\phi_{-}^{(0)}}^{\phi_{0}} d \phi \sqrt{2\left(f(\phi)-f\left(\phi_{-}^{(0)}\right)\right)}
$$

and the excess density $\phi_{e x c}^{(0)}=\frac{1}{2} \int_{0}^{\infty} d z\left[\phi(z)-\phi_{-}^{(0)}\right]$ at the surface can be calculated via

$$
\phi_{e x c}^{(0)}=\frac{1}{2} \int_{\phi_{-}^{(0)}}^{\phi_{0}} \frac{d \phi\left(\phi-\phi_{-}^{(0)}\right)}{\sqrt{2\left(f(\phi)-f\left(\phi_{-}^{(0)}\right)\right)}} .
$$

As long as $\mid f\left(\phi_{+}^{(0)}-f\left(\phi_{-}\right) \mid \ll\left(\phi_{+}^{(0)}-\phi_{-}\right)^{2} f^{\prime \prime}\left(\phi_{+}^{(0)}\right.\right.$, which is true for $\mu \ll 2$, the main contribution to this integral stems from $\phi$ values around $\phi_{+}^{(0)}$. The numerator in the integrand can then be expanded around $\phi_{+}^{(0)}$. Carrying this to second order and assuming $\mu \ll\left(\phi_{0}-\phi_{+}^{(0)}\right)$, one obtains

$$
\begin{array}{ll}
\phi_{\text {exc }}^{(0)} \approx \sqrt{\frac{1}{2}} \ln \left(\frac{2}{\phi_{+}^{(0)}-\phi_{0}}\right) & \left(\phi_{0}<\phi_{+}^{(0)}\right) \\
\phi_{\text {exc }}^{(0)} \approx \sqrt{\frac{1}{2}} \ln \left(\frac{4\left(\phi_{0}-\phi_{+}^{(0)}\right)}{\mu}\right) & \left(\phi_{0}>\phi_{+}^{(0)}\right) .
\end{array}
$$

Above the bulk demixing transition, the mixed film thus wets the wall at coexistence $(\mu \rightarrow 0)$ for $\phi_{0}>\phi_{+}^{(0)}$, and maintains a finite thickness for $\phi_{0}<\phi_{+}^{(0)}$. We will choose $\phi_{0}>\phi_{+}^{(0)}$ hereafter. From eqn. 2.11, one calculates the surface free energy to leading order in $\mu$ and $\left(\phi_{0}-\phi_{+}^{(0)}\right)$.

$$
F_{\text {exc }}^{(0)}=\frac{2 \sqrt{2}}{3}+\frac{1}{\sqrt{2}}\left(\phi_{0}-\phi_{+}^{(0)}\right)^{2}
$$

Below the bulk demixing transition, $\mu=\mu_{c}>0$ at coexistence and the thickness of the mixed film remains finite under all circumstances.

In the following, we shall first analyse the wetting behavior for the limiting cases where the order parameter 
varies varies on very short length scales $\left(\gamma / m_{+}^{* 2} \rightarrow 0\right)$ and on long length scales $\left(\gamma / m_{+}^{* 2} \rightarrow \infty\right)$ compared to the density. Then we will discuss the general case of intermediate $\gamma$, first analytically in an approximation where the potential (2.4) is replaced by a piecewise quadratic potential, and then numerically with the full potential (2.4).

\section{A. Limiting cases}

We consider first the wetting behavior at $\left(\gamma / m_{+}^{*}{ }^{2} \rightarrow\right.$ $0)$. In this case, $m$ adapts locally to $\phi$, and the order parameter profile $m(z)$ can be written as $m(\phi(z))$ with $m(\phi)=\theta+2 \kappa(\phi-1)$ for $\phi<1-\theta / 2 \kappa$ and $m(\phi)=0$ otherwise. Hence we are left with the effective one order parameter problem of calculating the density profile $\phi(z)$ in the slightly altered potential $\hat{f}(\phi)=f(m(\phi), \phi)$. Since $\hat{f}(\phi)$ is a smooth function with two minima, one can proceed as sketched above for the mixed film, with the analogous result: The demixed film wets the wall at $\phi>\phi_{+}^{*}$.

The analysis of the opposite case, $\left(\gamma / m_{+}^{* 2} \rightarrow \infty\right)$, is somewhat more involved. Here $\phi$ adapts locally to $m$; however, the bulk equation $\partial f / \partial \phi=\phi^{3}-\phi+\mu-\kappa m^{2}=0$ has two solutions $\phi_{ \pm}(m)$. One conveniently separates the profiles into four parts (I) - (IV) as indicated in Figure 1. The regions (I) and (III) are narrow slabs where $\phi(z)$ varies rapidly and $m$ can be approximated by a constant, $m=m_{0}$ at the surface (I) and $m_{2}$ at the interface (III). In (I), $\phi$ drops from it's surface value $\phi_{0}$ to the local equilibrium value $\phi_{+}\left(m_{0}\right)$, and in (III), it switches from $\phi_{+}\left(m_{2}\right)$ to $\phi_{-}\left(m_{2}\right)$. The other two regions, (II) and (IV), are much wider; The order parameter $m(z)$ varies slowly and $\phi(z)$ adjusts locally to $m(z)$, such that $\phi=\phi_{+}(m)$ in (II) and $\phi=\phi_{-}(m)$ in (IV).

To make the argument more quantitative, we specify the actual subdivision of the excess free energy of (2.1),

$$
\mathcal{F}_{\text {exc }}=f_{s}\left(m_{0}, \phi_{0}\right)+\mathcal{F}_{I}+\mathcal{F}_{I I}+\mathcal{F}_{I I I}+\mathcal{F}_{I V}
$$

with

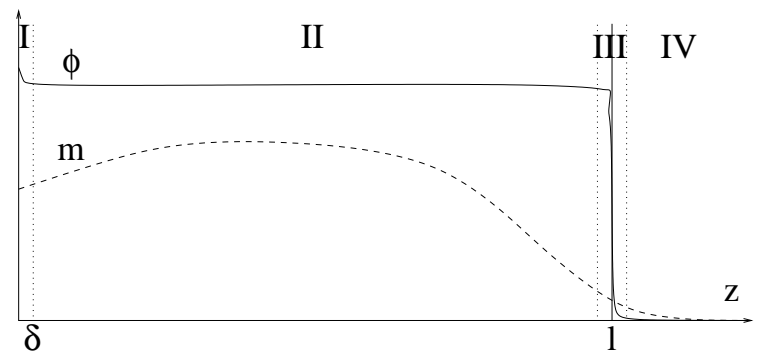

FIG. 1. Schematic sketch of density and order parameter profiles in the limit $\gamma / m_{+}^{* 2} \rightarrow \infty$. See text for more explanation.

$$
\begin{aligned}
\mathcal{F}_{I} & =\int_{0}^{\delta} d z\left[\frac{1}{2}\left(\frac{d \phi}{d z}\right)^{2}+f\left(m_{0}, \phi\right)-f\left(m_{0}, \phi_{+}\left(m_{0}\right)\right)\right] \\
\mathcal{F}_{I I} & =\int_{0}^{l} d z\left[\frac{1}{2} \gamma\left(\frac{d m}{d z}\right)^{2}+f\left(m, \phi_{+}(m)\right)\right] \\
\mathcal{F}_{I I I} & =\int_{l-\delta}^{l} d z\left[\frac{1}{2}\left(\frac{d \phi}{d z}\right)^{2}+f\left(m_{2}, \phi\right)-f\left(m_{2}, \phi_{+}\left(m_{2}\right)\right)\right] \\
& +\int_{l}^{l+\delta} d z\left[\frac{1}{2}\left(\frac{d \phi}{d z}\right)^{2}+f\left(m_{2}, \phi\right)-f\left(m_{2}, \phi_{-}\left(m_{2}\right)\right)\right] \\
\mathcal{F}_{I V} & =\int_{0}^{l} d z\left[\frac{1}{2} \gamma\left(\frac{d m}{d z}\right)^{2}+f\left(m, \phi_{-}(m)\right)\right] .
\end{aligned}
$$

The calculation for the regions (I), (III), and (IV) can proceed in an analogous way as sketched earlier for the mixed film: The profiles of $\phi(z)$ in (I), (III), and of $m(z)$ in (IV) are monotonic and the integration constant (cf. 2.10) is known (zero). One obtains to leading order in $\mu$ and $\left(\phi_{0}-\phi_{+}\left(m_{0}\right)\right)$

$$
\begin{aligned}
F_{I} & =\sqrt{\frac{1}{2}}\left(\phi_{0}-\phi_{+}\left(m_{0}\right)\right)^{2}+\cdots \\
F_{I I I} & =\frac{2 \sqrt{2}}{3}+\mathcal{O}\left(\left(\mu-\kappa m_{2}^{2}\right)^{2}\right) \\
F_{I V} & =\frac{m_{2}^{2}}{2} \sqrt{\gamma(4 \kappa+\kappa \mu-\theta)}
\end{aligned}
$$

In the region (II), the integration constant is unknown,

$$
\frac{1}{2} \gamma\left(\frac{d m}{d z}\right)^{2}-f\left(m, \phi_{+}(m)\right)=p,
$$

with $p>0$ if the profile of $m(z)$ is monotonic, and $p<0$ if $m(z)$ is nonmonotonic, like in Fig. 1. A connection between $p$ and the width $l$ of the film can be established using $l=\int_{m_{2}}^{m_{0}} d m /|d m / d z|$ in the first case, and

$$
l=\int_{m_{0}}^{m_{\max }} \frac{d m}{|d m / d z|}+\int_{m_{2}}^{m_{\max }} \frac{d m}{|d m / d z|}
$$

in the second case, where $m_{\max }$ solves $p=$ $-f\left(m_{\max }, \phi_{+}\left(m_{\max }\right)\right)$. Next we expand the function $f\left(m, \phi_{+}(m)\right)$ about it's minimum $m_{+}^{*}$, leading to

$$
f\left(m, \phi_{+}(m)\right) \approx\left(1-\kappa^{2}\right) m_{+}{ }^{4}\left(\left(\frac{m}{m_{+}^{*}}-1\right)^{2}-\frac{1}{4}\right)
$$

One deduces the characteristic length scale,

$$
\lambda=\sqrt{\frac{\gamma}{2\left(1-\kappa^{2}\right)}} \frac{1}{m_{+}^{*}},
$$

which grows very large in the limit $\gamma /{m_{+}^{*}}^{2} \rightarrow \infty$. The result for $\mathcal{F}_{I I}$ can therefore be expanded in powers of $e^{-l / \lambda}$. After adding up all contributions (I)-(IV) and minimizing with respect to $m_{2}$, the total excess free energy of the demixed film takes the form $F_{\text {exc }}=F_{\text {surf }}\left(m_{0}, \phi_{0}\right)+$ $F_{\text {int }}+V(l)$ with the surface contribution 


$$
\begin{aligned}
F_{\text {surf }}= & f_{s}\left(m_{0}, \phi_{0}\right)+\sqrt{\frac{1}{2}}\left(\phi_{0}-\phi_{+}\left(m_{0}\right)\right)^{2} \\
& +8 \lambda \mu_{c}\left(1-m_{0} / m_{+}^{*}\right)^{2},
\end{aligned}
$$

the interface contribution

$$
F_{\text {int }}=\frac{2 \sqrt{2}}{3}+8 \lambda \mu_{c},
$$

and a surface/interface interaction term

$$
\begin{aligned}
V(l)= & 2\left(\mu-\mu_{c}\right) l-32 \lambda \mu_{c}\left(1-m_{0} / m_{+}^{*}\right) e^{-l / \lambda} \\
& +16 \lambda \mu_{c}\left(A-\left(1-m_{0} / m_{+}^{*}\right)^{2} B\right) e^{-2 l / \lambda},
\end{aligned}
$$

where $B=\left[\lambda \sqrt{\gamma\left(\kappa-m_{+}^{* 2}\left(1-\kappa^{2}\right) / 4\right.}\right]^{-1}$, and $A=-3$ or $A=1$, depending on whether or not the profile $m(z)$ is monotonic.

The result can now be discussed. At $m_{0}<m_{+}^{*}$, the leading term $e^{-l / \lambda}$ of the potential $V(l)$ is attractive, and wetting is not possible. At $m_{0}>m_{+}^{*}$, an infinitely thick demixed film is metastable at coexistence. It's free energy difference to the mixed film $\Delta F=F_{e x c}-F_{e x c}^{(0)}$ is up to third order in $m_{+}^{*}$

$$
\begin{aligned}
\Delta F= & \frac{1}{\sqrt{2}}\left(\sqrt{\gamma\left(1-\kappa^{2}\right)}\left(1+\left(1-m_{0} / m_{+}^{*}\right)^{2}\right) m_{+}^{* 3}\right. \\
& \left.+\left(C_{m} / \sqrt{2}-\kappa\left(\phi_{0}-\phi_{+}\left(m_{0}\right)\right)\right) m_{0}^{2}\right)
\end{aligned}
$$

The limit $\gamma / m_{+}^{* 2} \rightarrow \infty$ can be taken in two ways: either $\gamma \rightarrow \infty$ at fixed $m_{+}^{*}$, or $m_{+}^{*} \rightarrow 0$ at fixed $\gamma$. In the first case the first term in eqn. $(2.26)$ dominates and the free energy of the demixed film exceeds that of the mixed film: The film remains mixed and dewets accordingly.

The second case is more subtle. Here, the second term dominates, and the free energy of the mixed film may be less favorable, depending on the ratio of $C_{m}$ and $\left(\phi_{0}-\phi_{+}^{*}\right)$. Note that the density enhancement at the surface, $\sqrt{2} \kappa\left(\phi_{0}-\phi_{+}^{*}\right)$, acts as an additional surface coupling, which opposes the effect of $C_{m}$. The parameter $C_{m}$ accounts for the direct reduction of interacting neighbours right at the surface. It is counterbalanced by the fact that the density $\phi_{0}$ close to the surface is higher than in the bulk. If the latter effect dominates, the film demixes at the surface even for $m_{+}^{*} \rightarrow 0$ or $T \rightarrow T_{c e p}$.

\section{B. Analytical results in a piecewise parabolic potential}

At fixed $m_{+}^{*}$, we have seen that the demixed film wets the substrate in the limit $\gamma \rightarrow 0$, where the order parameter $m$ varies much faster than the density $\phi$, and dewets at $\gamma \rightarrow \infty$, where the density varies much faster than the order parameter. Now we consider intermediate values of $\gamma$, where the two characteristic length scales become comparable. Far from the critical end point, this is the usual case in a binary liquid, since the interaction ranges responsible for liquid/gas separation and demixing are comparable.

In order to carry further the analytical analysis, we approximate the free energy density $f(\phi, m)$ (2.4) by a piecewise quadratic form

$$
f(\phi, m)=\frac{1}{2}(\phi-\tilde{\phi}, m-\tilde{m}) \tilde{\tilde{f}}\left(\begin{array}{c}
\phi-\tilde{\phi} \\
m-\tilde{m}
\end{array}\right)+\sigma \tilde{\mu},
$$

with three pieces corresponding to the gas phase and the two liquid phases, separated by the lines

$$
\phi_{\text {sep }}(m)=-\kappa\left(m^{2}+m_{+}^{* 2}-|m| m_{+}^{*}\right)+\tilde{\mu} / 2 .
$$

and $m \equiv 0$ at $\phi>\phi_{\text {sep }}(0)$. Here $\tilde{\mu}=\mu-\mu_{c}, \sigma=-1$ for $\phi>\phi_{\text {sep }}(m)$ (gas phase), $\sigma=+1$ for $\phi<\phi_{\text {sep }}(m)$ (liquid phases), and the parabolae are adjusted to the leading terms in the expansion of the functional (2.4) about its minima,

$$
\left(\begin{array}{c}
\tilde{\phi} \\
\tilde{m}
\end{array}\right)=\left(\begin{array}{c}
-1 \\
0
\end{array}\right), \quad \widetilde{\tilde{f}}=\left(\begin{array}{cc}
2 & 0 \\
0 & 4 \kappa
\end{array}\right),
$$

for $\phi<\phi_{\text {sep }}(m)$ (gas phase), and

$$
\left(\begin{array}{c}
\tilde{\phi} \\
\tilde{m}
\end{array}\right)=\left(\begin{array}{c}
1+\kappa m_{+}^{* 2} / 2 \\
\pm m_{+}^{*}
\end{array}\right), \quad \tilde{\widetilde{f}}=\left(\begin{array}{cc}
2 & \mp 2 \kappa m_{+}^{*} \\
\mp 2 \kappa m_{+}^{*} & 2 m_{+}^{* 2}
\end{array}\right) \text {, }
$$

for $\phi>\phi_{\text {sep }}(m)$ (liquid phases), where the upper sign holds for $m>0$, the lower for $m<0$. The choice (2.28) of $\phi_{\text {sep }}$ ensures that the potential $f(m, \phi)$ is continuous.

In such a potential, profiles of demixed films correspond to paths in the $(\phi, m)$ space which can be separated into three parts: (i) moving in one of the liquid regions from $\left(\phi_{0}, m_{0}\right)$ to $\left(\phi_{1}, m=0\right)$; (ii) following the edge $(m \equiv 0)$ between the two liquid regions from $\left(\phi_{1}, 0\right)$ to $\left(\phi_{\text {sep }}(0), 0\right)$; (iii) moving in the gas region from $\left(\phi_{\text {sep }}(0), 0\right)$ to $(-1,0)$. On principle, a direct transition from (i) to (iii) is conceivable. For the parameters $\phi_{0}$ of interest, however, such profiles turn out to be energetically less favorable than the profiles which have an intermediate (ii). Profiles of mixed films have two parts (ii) and (iii) only. We shall denote $l_{(i)} \equiv l, l_{(i i)}$, and $l_{(i i i)}$, the length of the slab spent in region (i), (ii) or (iii), respectively.

At given slab length and boundary conditions, the free energy in each of the slabs can be calculated exactly using

$$
\begin{aligned}
& \int_{0}^{l} d z \frac{1}{2}\left\{\left(\frac{d u}{d z}\right)^{2}+\frac{u^{2}}{\lambda^{2}}\right\} \\
& =\frac{1}{4}\left\{(u(0)+u(l))^{2} \tanh \frac{l}{2 \lambda}+(u(0)-u(l))^{2} \operatorname{coth} \frac{l}{2 \lambda}\right\} .
\end{aligned}
$$

The calculation is straightforward in the regimes (ii) and (iii). In (i), the free energy functional has to be diagonalized first:

$$
\mathcal{F}_{(i)}=\frac{1}{2} \int_{0}^{l} d z\left\{\left[\left(\frac{d v}{d z}\right)^{2}+\frac{v^{2}}{\lambda_{1}^{2}}\right]+\left[\left(\frac{d w}{d z}\right)^{2}+\frac{w^{2}}{\lambda_{2}^{2}}\right]\right\}
$$


with

$$
\begin{aligned}
\lambda_{1,2}^{-2} & =1+\frac{m_{+}^{* 2}}{\gamma} \mp \sqrt{\left(\frac{m_{+}^{*}{ }^{2}}{\gamma}\right)^{2}+\frac{m_{+}^{* 2}}{\gamma}\left(4 \kappa^{2}-2\right)+1,} \\
\left(\begin{array}{c}
v \\
w
\end{array}\right) & =\frac{1}{\sqrt{e^{\delta}+e^{-\delta}}}\left(\begin{array}{cc}
e^{-\delta / 2} & e^{\delta / 2} \\
e^{\delta / 2} & -e^{-\delta / 2}
\end{array}\right)\left(\begin{array}{c}
\phi-\tilde{\phi} \\
\sqrt{\gamma}(m-\tilde{m})
\end{array}\right),
\end{aligned}
$$

where we have defined

$$
\delta=\frac{1}{2} \ln \left(\frac{2-\lambda_{1}^{-2}}{\lambda_{2}^{-2}-1}\right) .
$$

The parameter $\delta$ or alternatively $\gamma / m_{+}^{* 2}$ determines the wetting behavior. Fig. 2 shows the two length scales $\lambda_{1}$ and $\lambda_{2}$ as a function of $\gamma / m_{+}^{*}$. The length $\lambda_{1}$ is always larger than $\lambda_{2}$. At $\gamma / m_{+}^{*} \gg 1$ or $\delta \gg 0$, it characterizes the spatial variations of $m(z)$ and grows linearly with $\gamma / m_{+}^{* 2}$; at $\gamma / m_{+}^{* 2} \ll 1$ or $\delta \ll 0$, it characterizes the variations of $\phi(z)$ and remains largely independent of $\gamma / m_{+}^{*}$. These are the limiting regimes discussed in the previous subsection. At $\gamma / m_{+}^{* 2} \approx 1$ or $\delta \approx 0$, both $\lambda_{1}$ and $\lambda_{2}$ are related to linear combinations of $\phi(z)$ and $m(z)$.

The further calculation proceeds as follows: The free energy in (iii) is given by

$$
F_{(i)}=\sqrt{2} / 2\left(1+\left(\phi_{s e p}(0)+1\right)^{2}\right) .
$$

In the region (ii), the result for the free energy is expanded in powers of $e^{-\sqrt{2} l_{(i i)}}$ up to the second order and minimized with respect to $l_{(i i)}$. The free energy calculated in (i) is expanded up to second order in powers of $e^{-l / \lambda_{1}}$ and up to first order in $e^{-l / \lambda_{2}}$, where $l \equiv l_{(i)}$. The three contributions are then added up, and the sum is minimized with respect to $\phi_{1}$ and $m_{0}$ at given surface coupling $C_{m}$. The solution has to be compared with the free energy of a mixed film, which is calculated analogously.

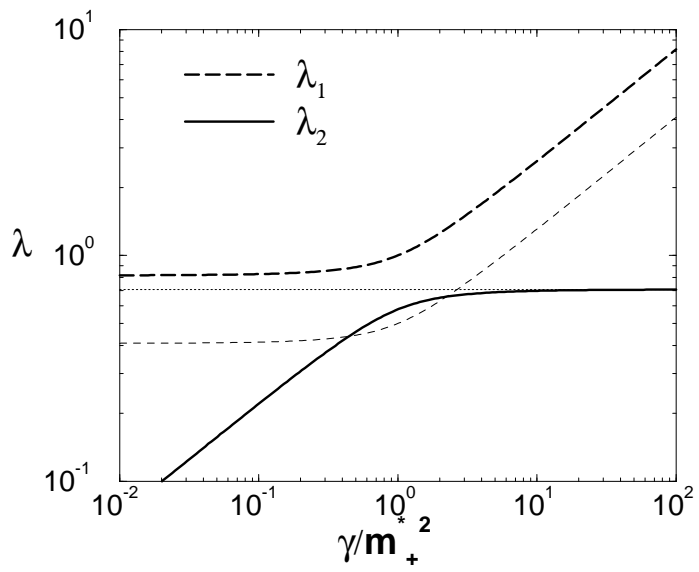

FIG. 2. Length scales $\lambda_{1}$ and $\lambda_{2}$ vs. $\gamma / m_{+}^{* 2}$ at $\kappa=0.5$. Thin line shows $\lambda_{1} / 2$ for comparison.
We only report the result for the case $C_{m}=0$ here. The expressions obtained for arbitrary $C_{m}$ are more complicated, but qualitatively similar. Without loss of generality, we can assume $m>0$ in the demixed film. As long as $m_{0}>0$, the surface order parameter $m_{0}$ and the free energy difference $\Delta F$ between the mixed and demixed film can then be expanded as

$$
\begin{aligned}
& \sqrt{\gamma} m_{0}=\sqrt{\gamma} m_{+}^{*}+\iota_{0}+\iota_{1} e^{-l / \lambda_{1}}+\iota_{2} e^{-l / \lambda_{2}}+\iota_{3} e^{-2 l / \lambda_{1}} \\
& \Delta F(l)=\Delta F_{(i i)}+\tau_{0}+\tau_{2} e^{-l / \lambda_{1}}+\tau_{2} e^{-l / \lambda_{2}}+\tau_{3} e^{-2 l / \lambda_{1}}
\end{aligned}
$$

Using the abbreviations

$$
K_{0}=\frac{e^{\delta}+e^{-\delta}}{\lambda_{1} \lambda_{2}}, \quad \text { and } \quad K_{ \pm}=\frac{\lambda_{1} \lambda_{2}}{e^{ \pm \delta} \lambda_{1}+e^{\mp \delta} \lambda_{2}}
$$

the coefficients can be written as

$$
\begin{gathered}
\iota_{0}=\left(\lambda_{2}^{-1}-\lambda_{1}^{-1}\right) K_{-} \cdot\left(\phi_{0}-\tilde{\phi}\right) \\
\iota_{1,2}=-2 K_{0} K_{+} K_{-} e^{ \pm \delta} \cdot \sqrt{\gamma} m_{+}^{*} \\
\iota_{3}=-2 K_{0} K_{-}^{2}\left(1+8 K_{+} e^{-\delta} / \lambda_{1}\right) \cdot\left(\phi_{0}-\tilde{\phi}\right) \\
\tau_{0}=K_{0}\left(K_{+} \cdot \gamma m_{+}^{* 2}+K_{-} \cdot\left(\phi_{0}-\tilde{\phi}\right)^{2}\right) \\
\tau_{1,2}= \pm 2 K_{0} K_{+} K_{-} / \lambda_{2,1} \cdot \sqrt{\gamma} m_{+}^{*} \cdot\left(\phi_{0}-\tilde{\phi}\right) \\
\tau_{3}=K_{0} K_{-} K_{+}^{2} e^{\delta}\left(e^{-\delta} / \lambda_{2}-e^{\delta} / \lambda_{1}\right) / \lambda_{2} \cdot \gamma m_{+}^{*} \\
+K_{0} K_{-}^{2} e^{-\delta}\left(1+8 K_{+} e^{-\delta} / \lambda_{1}\right) / \lambda_{2} \cdot\left(\phi_{0}-\tilde{\phi}\right)^{2},
\end{gathered}
$$

and with $h=m_{+}^{*}\left(1-\kappa^{2}\right)-2 \tilde{\mu}, p=\phi_{0}-\tilde{\phi}+\kappa m_{+}^{* 2}$,

$$
\Delta F_{(i i)}=-\frac{1}{\sqrt{2}} p^{2}-\frac{h}{2 \sqrt{2}}\left(1+\ln \frac{4 p^{2}}{h}\right) .
$$

When taking the limits $\delta \rightarrow \pm \infty$, one recovers qualitatively [23] the behavior discussed in the previous section.

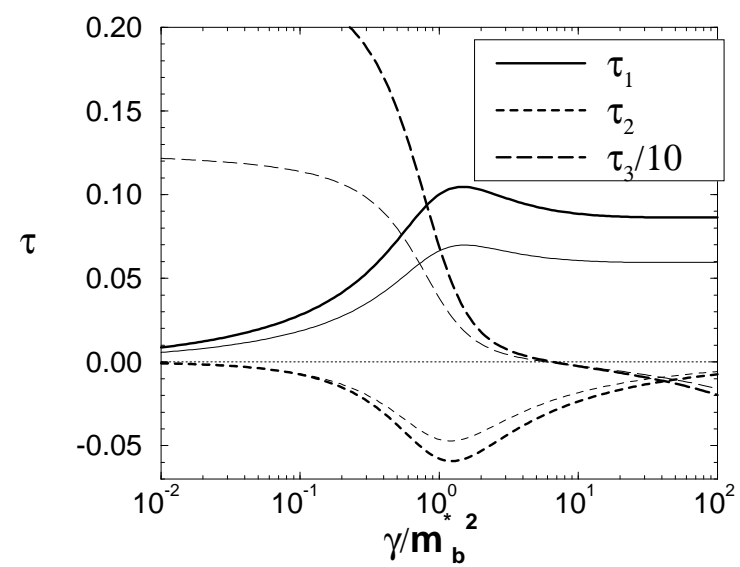

FIG. 3. Coefficients $\tau_{i}$ of interfacial potential vs. $\gamma / m_{+}^{* 2}$ for $\kappa=0.5, \Phi_{0}=1.5$, and surface coupling $C_{m}=0$. (thick lines) and $C_{m}=0.2$ (thin lines). 
The function $\Delta F(l)$ can be conceived as an effective interface potential for the demixed film. The parameters $\tau_{i}$ for a choice of $\phi_{0}\left(\phi_{0}=1.5\right)$ and two values of $C_{m}$, $C_{m}=0$ according to (2.37) and $C_{m}=0.02$, are shown in Fig. 3. One finds that $\tau_{1}$ is always positive, $\tau_{2}$ is always negative, and $\tau_{3}$ changes sign from positive to negative as $\gamma / m_{+}^{*}$ increases. The leading term of the potential $F(l)$ is thus positive, and one expects a first order wetting transition and a prewetting line. On the other hand, the expansions 2.35) are only valid as long as the surface order parameter $m_{0}$ is positive. According to eqn. (2.36), the coefficients $\iota_{i}$ of the expansion for $m_{0}(l)$ are negative except for the zeroth order term $\iota_{0}$. Hence $m_{0}$ decreases with film thickness and may vanish at some thickness $l_{c}$. In this case, the film mixes continuously at $l_{c}$, and the prewetting line turns into a second order demixing line sufficiently far from coexistence.

\section{Numerical solution}

The analytical results of the previous subsection provided insight into the competition of length scales in the binary fluid and the wetting scenarios which can be expected on a wall as a result. However, a reliable calculation of actual phase diagrams, including the details of the prewetting line, is not possible on the basis of the expansion (2.35). We have thus supplemented the analytical work by a numerical minimization of the functional (2.1) in the $\mu-\phi_{0}$ plane for selected sets of parameters $\gamma$ and $C_{m}$.

The problem is simplified considerably due to the fact that $\phi(z)$ is a monotonic function of $z$, i.e., $m(z)$ can be expressed as a function $m(\phi)$. The bulk free energy functional in (2.1) can thus be rewritten as

$$
\begin{aligned}
\mathcal{F} & =\int_{0}^{\infty} d z\left\{\frac{1}{2}\left(1+\gamma\left(\frac{d m}{d \phi}\right)^{2}\right)\left(\frac{d \phi}{d z}\right)^{2}+f(m(\phi), \phi)\right\} \\
& =\int_{\phi_{-}^{*}}^{\phi_{0}} d \phi \sqrt{1+\gamma\left(\frac{d m}{d \phi}\right)^{2}} \sqrt{f(m(\phi), \phi)-f\left(0, \phi_{-}^{*}\right)},
\end{aligned}
$$

where the integration constant (2.10) has been identified and exploited as usual. Minimization with respect to the function $m(\phi)$ leads to the Euler-Lagrange equation

$$
2 \gamma f(m, \phi) \frac{d^{2} m}{d \phi^{2}}=\left(1+\gamma\left(\frac{d m}{d \phi}\right)^{2}\right)\left(\frac{\partial f}{\partial m}-\gamma \frac{d m}{d \phi} \frac{\partial f}{\partial \phi}\right),
$$

which we have solved using the Verlet algorithm.

Some results are shown in Figs. 4, 5, 6, and 0. As anticipated in the previous subsection, we find a first order wetting transition, a discontinuous prewetting line and a continuous demixing line. At surface coupling $C_{m}>0$, the demixing line joins the prewetting line in a surface critical end point (Fig. (1). The prewetting line separates a demixed thick film from a mixed thin film (see profiles

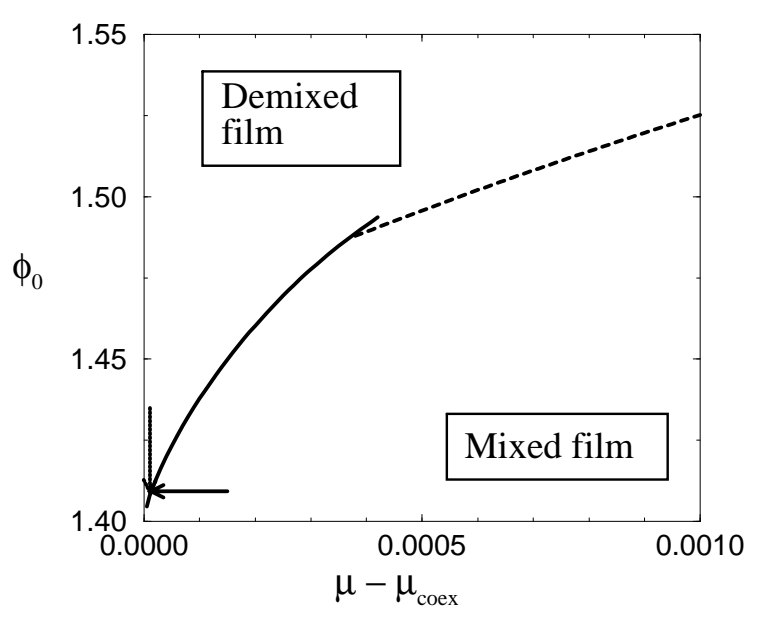

FIG. 4. Phase diagram in the $\mu-\phi_{0}$ plane. Parameters are $\kappa=0.5, \theta=0.1$ and $\gamma=1 ., C_{m}=0.2$. Solid line indicates first order transition, dashed line second order transition.

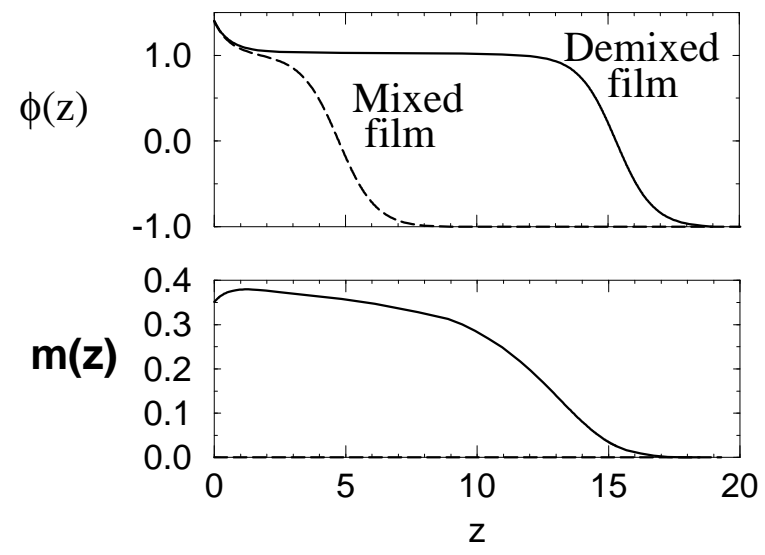

FIG. 5. Density and order parameter profiles for the coexisting mixed and demixed film at the point in the $\mu-\phi_{0}$ plane indicated by the arrows in Fig. 6 .

in Fig. 5) before reaching the critical end point, then two demixed films of different thickness, and finally vanishes in a critical point. On decreasing the surface coupling $C_{m}$, the critical end point and the critical point move closer to each other, until they merge in a surface tricritical point.

Fig. 6 shows two cases of phase diagrams in the $\phi_{0}-\mu$ plane for $C_{m}=0$ and two different $\gamma$ at fixed $\theta$, i. e., at fixed bulk order parameter $m_{+}^{*}$. With increasing $\gamma$, the prewetting line shifts towards larger $\phi_{0}$ and extends deeper into the off-coexistence region. As $\gamma \rightarrow \infty$, it moves to $\phi_{0} \rightarrow \infty$, the film remains mixed and thin at all finite $\phi_{0}$. At $\gamma \rightarrow 0$, on the other hand, the line becomes flat, approaches $\phi_{+}^{*}$, and the tricritical point where it turns into a second order line moves to $\mu_{t} \rightarrow \mu_{c}$. The numerical results thus agree with the conclusions from section II A. 
Fig. 7 demonstrates what happens if instead of making $\gamma$ larger, one increases the characteristic length scale of order parameter fluctuations by decreasing $\theta$, i.e., approaching the critical end point (reducing $m_{+}^{*}$ ). Far from liquid/vapour coexistence, the transition line still moves towards larger $\phi_{0}$. However, the effect reverses close to coexistence, the demixing transition is now shifted to lower surface densities $\phi_{0}$. Furthermore, the length of the prewetting line shrinks instead of growing.

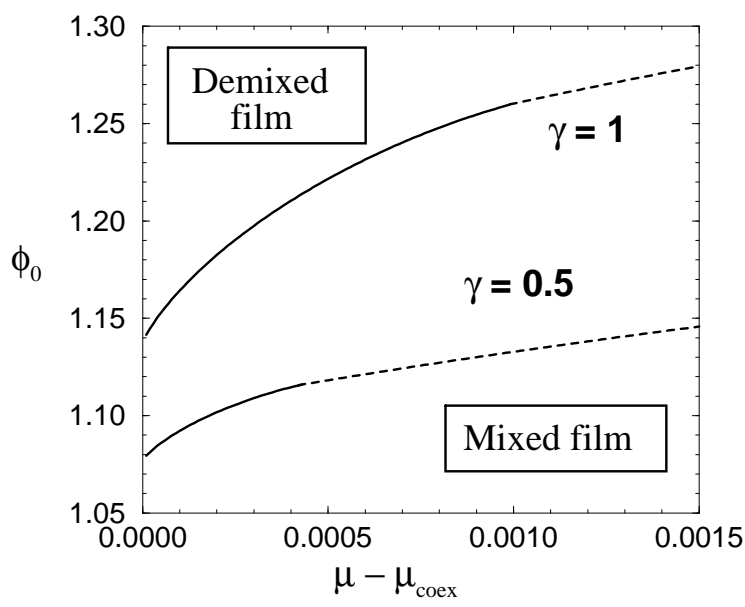

FIG. 6. Phase diagrams in the $\mu-\phi_{0}$ plane for different $\gamma$ with $\theta=0.1$, surface coupling $C_{m}=0$, and parameters as in Fig. 6 otherwise. Solid lines indicate first order transition, dashed lines second order transitions.

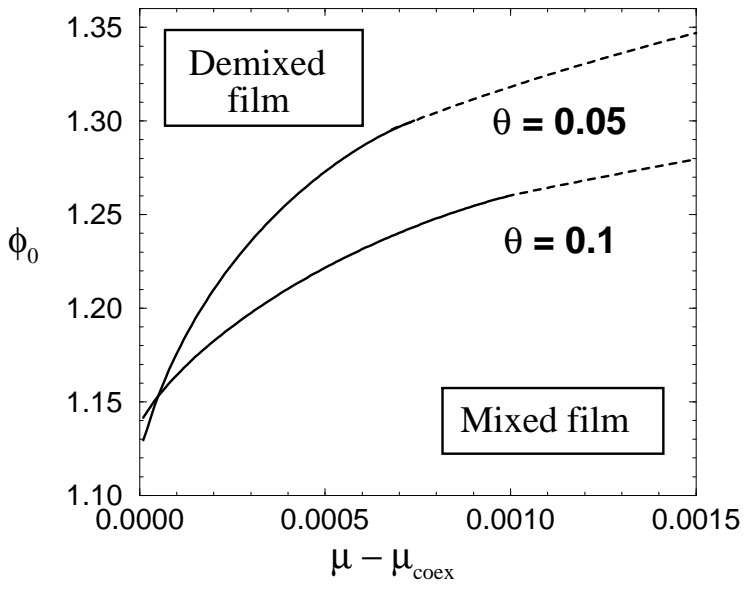

FIG. 7. Phase diagrams in the $\mu-\phi_{0}$ plane for different $\theta$ with $\gamma=1$., surface coupling $C_{m}=0$. and parameters as in Fig. 6 otherwise. Solid lines indicate first order transition, dashed lines second order transitions.

\section{MONTE CARLO SIMULATIONS}

In this section we describe Monte Carlo simulation studies of the subcritical wetting behaviour of a symmetrical binary fluid at a structureless wall.

\section{A. Model and simulation details}

The system we have studied is a symmetrical binary fluid, having interparticle interactions of the LennardJones (LJ) form:

$$
u\left(r_{i j}\right)=4 \epsilon_{i j}\left[\left(\frac{\sigma_{i j}}{r_{i j}}\right)^{12}-\left(\frac{\sigma_{i j}}{r_{i j}}\right)^{6}\right]
$$

We made the following choice of model parameters: $\sigma_{11}=\sigma_{22}=\sigma_{12}=\sigma=1 ; \epsilon_{11}=\epsilon_{22}=\epsilon ; \epsilon_{12}=0.7 \epsilon$. i.e. interactions between similar species are treated identically, but those between unlike species are weakened. The inter-particle potential was truncated at a distance of $R_{c}=2.5 \sigma$ and no long-range correction or potential shift was applied.

The fluid was confined within a cuboidal simulation cell having dimensions $P_{x} \times P_{y} \times D$, in the $x, y$ and $z$ coordinate directions respectively, with $P_{x}=P_{y} \equiv P$. The simulation cell was divided into cubic sub-cells (of size the cutoff $R_{c}$ ) in order to aid identification of particle interactions. Thus $P=p R_{c}$ and $D=d R_{c}$, with $p$ and $d$ both integers. To approximate a semi-infinite geometry, periodic boundary conditions were applied in the $x$ and $y$ directions, while hard walls were applied in the unique $z$ direction at $z=0$ and $z=D$. The hard wall at $z=0$ was made attractive, using a potential designed to mimic the long-ranged dispersion forces between the wall and the fluid 24]:

$$
V(z)=\epsilon_{w}\left[\frac{2}{15}\left(\frac{\sigma_{w}}{z}\right)^{9}-\left(\frac{\sigma_{w}}{z}\right)^{3}\right]
$$

Here $z$ measures the perpendicular distance from the wall, $\epsilon_{w}$ is a 'well-depth' controlling the interaction strength, and we set $\sigma_{w}=1$. No cutoff was employed and the wall potential was made to act equally on both particle species.

Monte-Carlo simulations of this system were performed using a Metropolis algorithm within the grand canonical $(\mu, V, T)$ ensemble [25]. Three types of Monte-Carlo moves were employed:

\section{Particle displacements}

2. Particle insertions and deletions

3. Particle identity swaps: $1 \rightarrow 2$ or $2 \rightarrow 1$

To maintain the symmetry of the model, the chemical potentials $\mu_{1}$ and $\mu_{2}$ of the two components were set equal 
at all times. Thus only one free parameter, $\mu=\mu_{1}=\mu_{2}$, couples to the overall number density $\rho=\left(N_{1}+N_{2}\right) / V$. The other variables used to explore the wetting phase diagram were the reduced well depth $\epsilon / k_{B} T$ and the reduced wall potential $\epsilon_{w} / k_{B} T$. During the simulations, the observables monitored were the total particle density profile

$$
\rho(z)=\left[N_{1}(z)+N_{2}(z)\right] / P^{2}
$$

the number difference order parameter profile,

$$
n(z)=\left|N_{1}(z)-N_{2}(z)\right| / P^{2}
$$

These profiles was accumulated in the form of a histogram. Other observables monitored were the total interparticle energy and the wall interaction energy.

The choice of system size was, as ever, a compromise between minimising finite-size effects and maximising computational throughput. Tests showed the profiles to be largely insensitive to the size of the wall area and hence $p=7$ was used, this being the largest computationally tractable size consistent with the necessary choice of the slit width $d$. The latter must clearly be considerably larger than the film thicknesses of interest in order to prevent the liquid film directly interacting with the hard wall at $z=D$. In the results presented below, the typical slit width used was $d=16$, corresponding to some 40 molecular diameters. For thin films a narrower slit of width $d=8$ was used.

\section{B. Wetting behaviour along a subcritical isotherm}

Accurate knowledge of bulk phase behaviour is an essential prerequisite for detailed studies of nearcoexistence wetting properties. In the present model, the locus of the liquid vapour coexistence curve and location

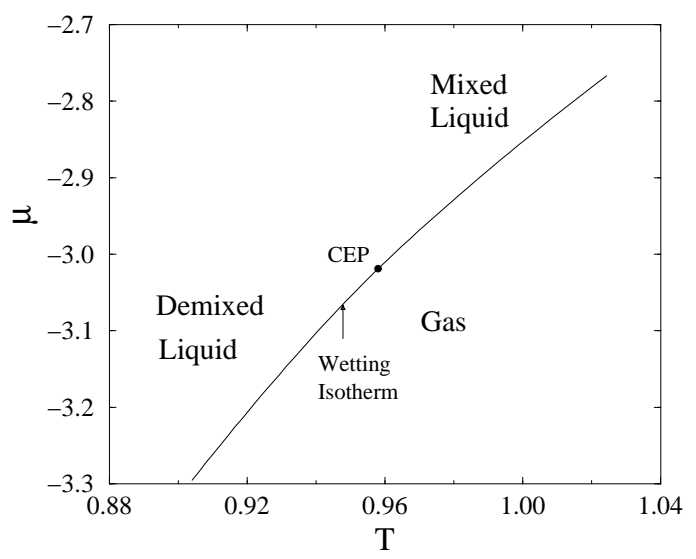

FIG. 8. The phase diagram in the $\mu-T$ plane of the symmetrical binary Lennard-Jones fluid model described in the text. Also shown is the location of the critical end point and the isotherm along which the wetting properties were studied of the critical end point are already known to high precision from a previous MC simulation study 9,5]. The phase diagram in the $\mu$-T plane (in standard LennardJones reduced units [25]) is shown in fig. 8. The critical end point is located at $T_{c e p}=0.958(3), \mu_{c e p}=-3.017(3)$ [9,5]. We note that although the locus of the coexistence curve is known to five significant figures, the position of the CEP along this tightly determined line is known only to three significant figures.

To determine the wetting properties at temperatures below $T_{c e p}$, the number density profile $\rho(z)$ was studied along the isotherm $T=0.9467$ as coexistence was approached from the vapour side. To achieve this, the chemical potential was incremented up to its coexistence value $\mu_{c x}(T)$ in a sequence of $6-10$ steps of constant size $\Delta \mu=0.0025$. This procedure was repeated for a number of different values of the wall-fluid potential strength $\epsilon_{w}$, allowing the influence of this parameter on the wetting behaviour to be ascertained. In all, six values of the $\epsilon_{w}$ were studied $\left(\epsilon_{w}=1.0,1.7,1.75,2.0,3.0,4.0\right)$. We describe the wetting behaviour for each in turn.

For $\epsilon_{w}=1.0$, fig. 9 shows that although the film thickness grows very slightly as coexistence is approached, it never exceed two molecular diameters. At no point in the profile does the density attain that of the liquid phase ( $\rho \approx 0.6)$. The presence of a thin wetting layer right up to coexistence implies incomplete (partial) wetting.

Increasing the wall potential to $\epsilon_{w}=1.70$ [fig. 10], results in considerably more structure near the wall compared to $\epsilon_{w}=1.0$, with clear density oscillations arising from excluded-volume 'packing effects' 27]. The profile is much more responsive to changes in the chemical potential and reaches a thickness of 4-5 molecular diameters close to coexistence.

For $\epsilon_{w}=1.75$, however, the situation changes qualitatively, as shown in fig. 11. On increasing the chemical potential, a clear jump is observed in both the thickness of the film, and the value of its density. In the thick film, the density of a significant portion of the film is that of

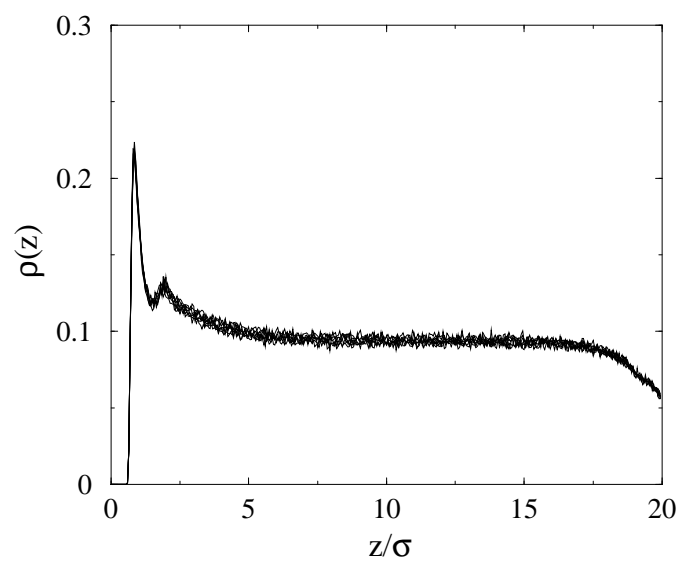

FIG. 9. Density profiles for $\epsilon_{w}=1.0$. Data are shown for 7 values of $\mu-\mu_{c x}$ in the range $[0,-1.5]$ 


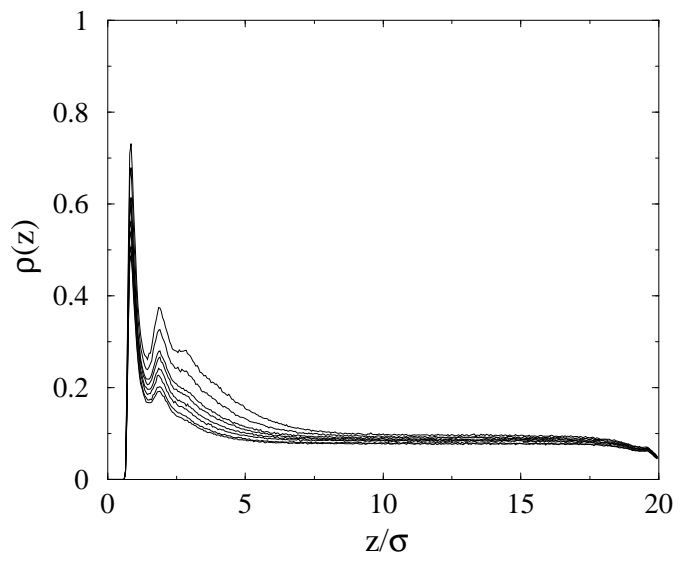

FIG. 10. Density profiles for $\epsilon_{w}=1.7$. Data are shown for 8 values of $\mu-\mu_{c x}$ in the range $[0,-1.525]$

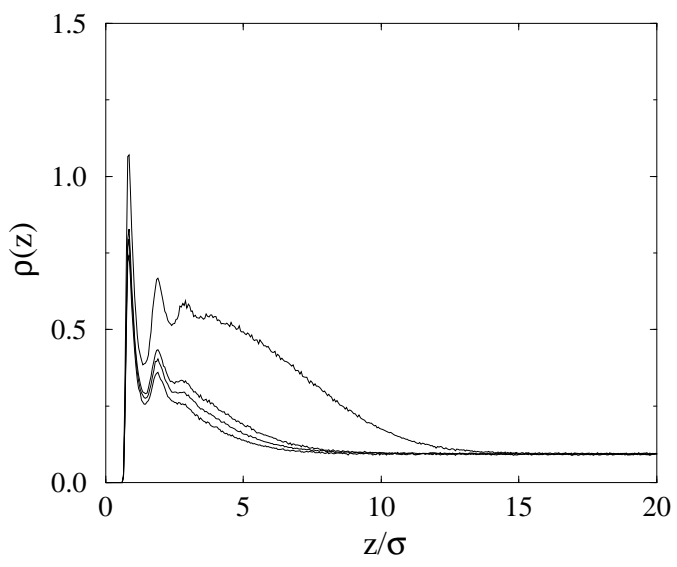

FIG. 11. Density profiles for $\epsilon_{w}=1.75$. Data are shown for 6 values of $\mu-\mu_{c x}$ in the range $[-0.025,-1.5]$

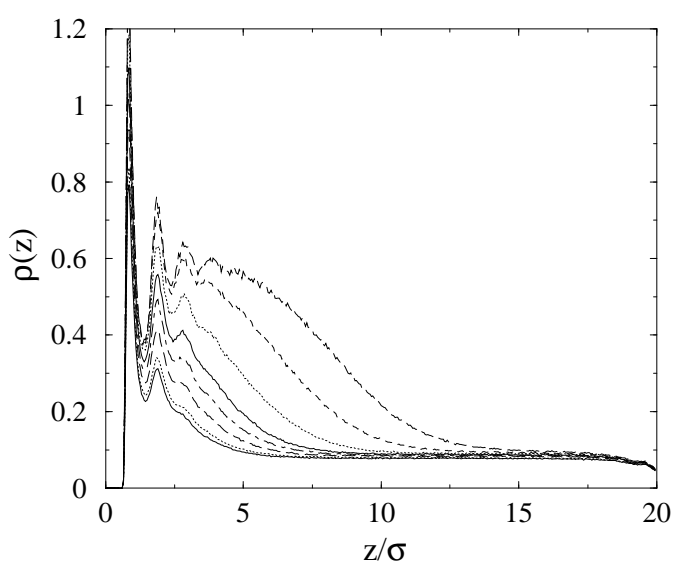

FIG. 12. Density profiles for $\epsilon_{w}=2.0$. Data are shown for 8 values of $\mu-\mu_{c x}$ in the range $[-0.025,-1.6]$ the bulk liquid. This thin-thick jump constitutes a prewetting transition, as previously observed in simulation studies of lattice gas models [26], Lennard-Jones fluids [13, 14, 27,28] as well as experimentally [29].

As the wall potential is increased to $\epsilon_{w}=2.0$ (fig. 12), the sharp prewetting transition is lost and instead the film thickness increases smoothly as $\mu$ approaches its coexistence value. This suggests that here the system is above the prewetting critical point ( 26$]$ ).

On increasing $\epsilon_{w}$ to 3.0, a new feature emerges (fig. 13(a)). As the chemical potential increases, the thickness of the film initially increases smoothly with increasing $\mu$. However, once the thickness reaches some 10 molecular diameters, a large jump occurs to a thickness of about 15 molecular diameters. Concomitant with this jump is a demixing of the film as a whole, as seen in the order parameter profile fig. 13 (b). The size of the jump in the layer thickness appears to reduce as the wall strength is increased to $\epsilon_{w}=4.0$ (fig. 14), suggesting a weakening of the transition.
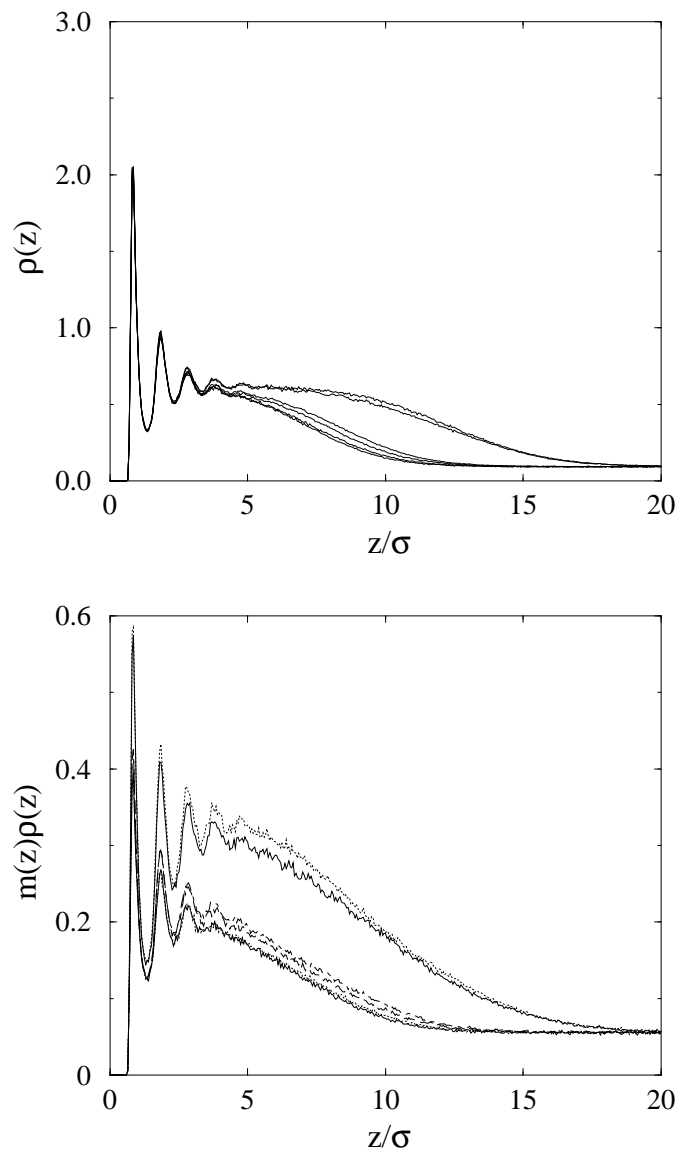

FIG. 13. (a) Density profiles for $\epsilon_{w}=3.0$. Data are shown for 6 values of $\mu-\mu_{c x}$ in the range $[-0.025,-1.55]$

(b) The corresponding order parameter profiles $n(z)$. 


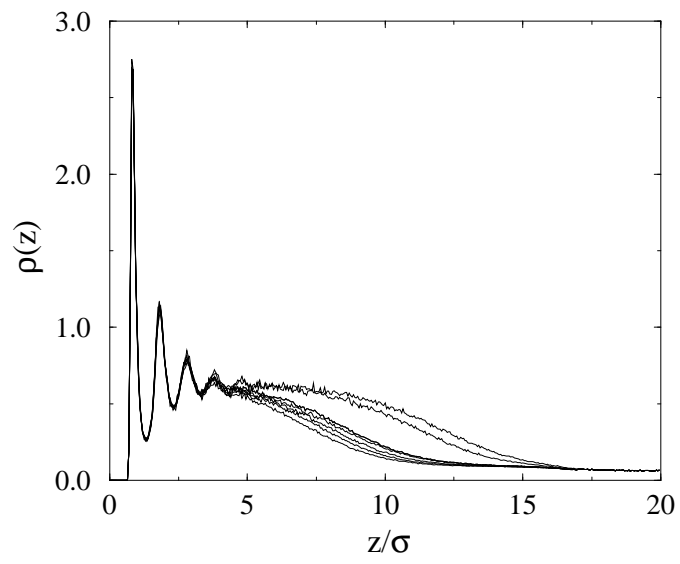

FIG. 14. Density profiles for $\epsilon_{w}=4$ 4.0. Data are shown for 8 values of $\mu-\mu_{c x}$ in the range $[-0.025,-1.6]$

\section{DISCUSSION}

The Monte Carlo simulation results at subcritical temperatures provide evidence that the mean field calculations correctly identify the qualitative wetting behaviour. They show that depending on the fluid-wall interaction strength $\epsilon_{w}$, a number of different wetting scenarios occur as liquid-vapour coexistence is approached from the vapour side. At small $\epsilon_{w}$, only a very thin film builds up on the wall. For intermediate values of $\epsilon_{w}$, a first prewetting transition is observed from a thin mixed film to a thick liquidlike mixed layer. Further increasing $\epsilon_{w}$ induces a second prewetting transition between a mixed liquidlike layer and a thicker demixed film, the situation being very similar to that shown in figure 5. The abrupt, first order, character of this latter transition appears to weaken on further increasing $\epsilon_{w}$, in accord with the theoretical predictions.

We will now attempt to set our results within the context of the bulk phase diagram of the binary liquid. To this end, we discuss the possible wetting scenarios in the vicinity of the critical end point $T_{c e p}$. As previously argued in the introduction, for temperatures $T<T_{\text {cep }}$ sufficiently close to $T_{c e p}$, the bulk correlation length $\xi$ of the demixed liquid is larger than the thickness $l_{*}$ of a mixed liquid layer at the wall. The state of order of the film thus depends strongly on the boundary conditions of the two interfaces confining the liquid layer. The nonselective liquid-vapor interface always favors mixing due to the reduced number of interacting neighbors in the interfacial region. The liquid-substrate interface, on the other hand, can either favour mixing or demixing depending on the strength of the fluid-wall potential. For a weakly attractive wall potential, mixing is favoured because the particle density at the wall is low and the presence of the wall reduces the number of interacting neighbours. For a strongly attractive wall, however, the high density at the wall can counteract the missing neighbour effect leading to an overall demixing tendency.
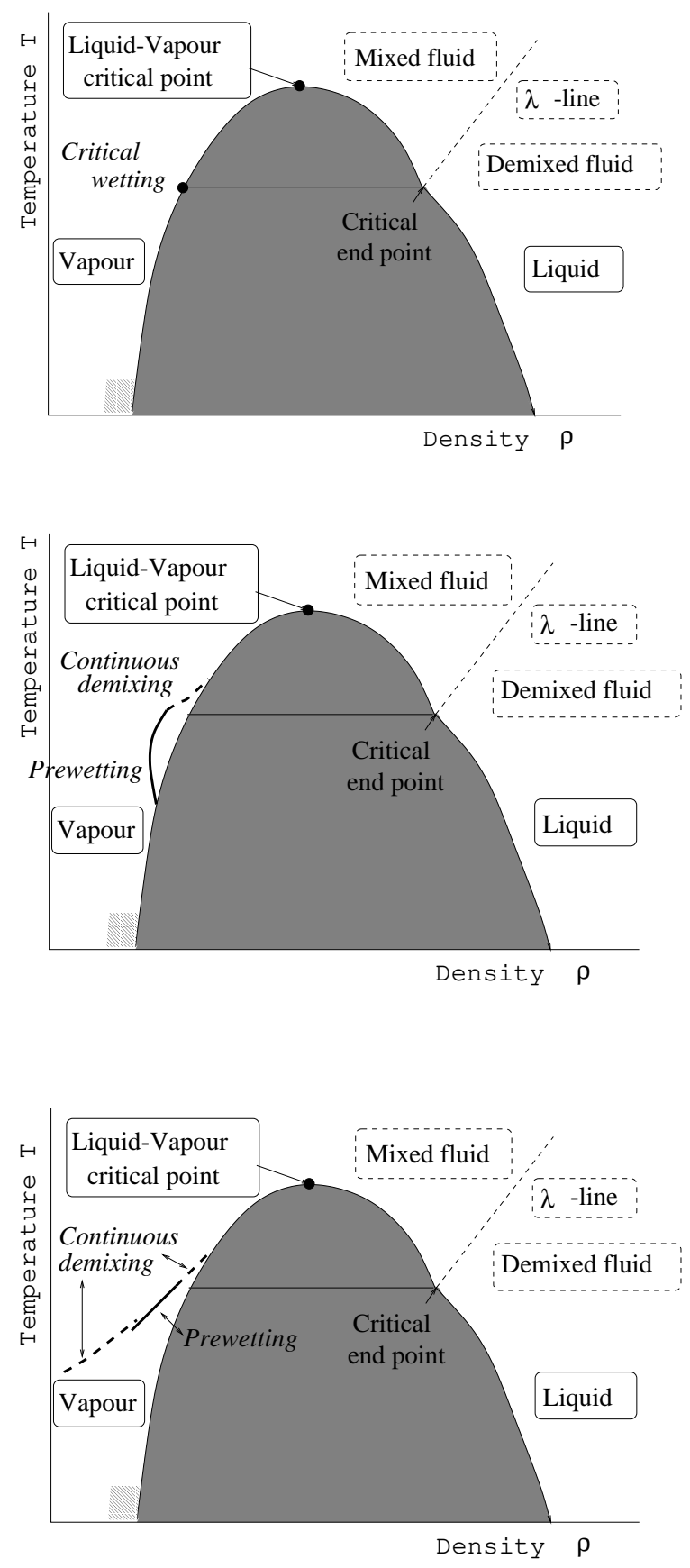

FIG. 15. Some possible schematic wetting phase diagrams in the temperature-density plane. (a) Weakly attractive wall: Critical end point $T_{c e p}$ of the $\lambda$-line is a critical wetting point, below which the wall is not wetted by the liquid. (b) Intermediate attraction: Demixing induced first order wetting transition at $T<T_{c e p}$ with prewetting line which evolves into a second order demixing line. (c) Strong attraction: Complete wetting at coexistence everywhere, but detached prewetting line or continuous demixing transitions off coexistence (in films of finite thickness). Hatched area indicates the possibility of conventional wetting transitions at lower temperatures. 
If the net effect favours mixing at the wall, a continuous demixing of the layer as coexistence is approached can be excluded. A first order transition involving a discontinuous increase of the film thickness upon demixing is still conceivable. However, we have shown in section II A, that (at the mean field level, at least) the demixed wetting film has a higher free energy than the corresponding mixed film provided the correlation length of composition fluctuations is sufficiently large.

At walls which suppress demixing, the film is thus always mixed close to the critical end point, and its thickness $l_{*}$ below the critical end point is finite. Hence the critical end point is automatically a critical wetting point. The resulting phase diagram is shown schematically in fig. 15 (a). Note that the wetting transition here is pinned by a bulk phase transition, a situation somewhat reminiscent of triple-point wetting [30,11].

The situation changes if the substrate favours demixing. In this situation, one component segregates to the surface of the film already slightly above $T_{c e p}$, and the order propagates continuously into the bulk of the film at $T_{\text {cep }}$. The film remains wet at $T_{c e p}$. From the results of section IIC (in particular, Fig. 7), one can deduce two possible scenarios. The film may still exhibit a first order wetting transition to a nonwet state at a temperature below $T_{c e p}$ (e.g. in Fig. 7) at $\phi_{0}=1.14$ ). The discontinuous phase transition at liquid/vapour coexistence then spawns a prewetting line which eventually switches into a second order demixing line and loops around the critical end point as suggested in Fig. 15 (b). If the wall is strongly attractive (e.g. at $\phi_{0}=1.27$ ) in Fig. 7), the wall wets at all temperatures, the prewetting line detaches from the coexistence line and is continued by second order demixing lines both at the high and low temperature side as sketched in Fig. 15 (c).

\section{Acknowledgements}

NBW thanks the Royal Society of Edinburgh, the EPSRC (grant no. GR/L91412) and the British Council for financial support.

[1] L.D. Gelb, K.E. Gubbins, R. Radhakrishnan, M. Sliwinska-Bartkowiak, Rep. Prog. Phys. 62, 1573 (1999).

[2] For reviews on wetting see P. G. de Gennes, Rev. Mod. Phys. 57, 827 (1985); M. Schick, in Les Houches, Session XLVIII - Liquids at Interfaces, J. Charvolin, J. F. Joanny, and J. Zinn-Justin eds. (Elsevier Science Publishers B.V., 1990); and Ref. 10]

[3] M. N. Barber, in Phase Transitions and Critical Phenomena, Vol. 8, p. 145, C. Domb, J. L. Lebowitz eds. (Academic Press, New York, 1983); V. Privman in Fi- nite Size Scaling and Numerical Simulation of Statistical Systems, V. Privman ed. (World Scientific, Singapore, 1990).

[4] M.P. Nightingale and J.O. Indekeu, Phys. Rev. Lett. 54, 1824 (1985); ibid 55, 1700 (1985); M. Krech, Phys. Rev. E 56, 1642 (1997); M. Krech, J. Phys.: Condens. Matter 11, R391 (1999).

[5] N. B. Wilding, M. Krech, Phys. Rev. E 57, 5795 (1998).

[6] A. Mukhopadhyay and B.M. Law, Phys. Rev. Lett. 83, 772 (1999).

[7] N. B. Wilding, F. Schmid, P. Nielaba, Phys. Rev. E 58, 2201 (1998).

[8] M. E. Fisher, P. J. Upton, Phys. Rev. Lett. 65, 2402 (1990); M. E. Fisher, M. C. Barbosa, Phys. Rev. B 43, 11177 (1991).

[9] N. B. Wilding, Phys. Rev. Lett. 78, 1488 (1997); N. B. Wilding, Phys. Rev. E55, E55, 6624 (1997).

[10] S. Dietrich in Phase Transitions and Critical Phenomena, Vol. 12, p. 1, C. Domb and J. L. Lebowitz eds (Academic Press, New York, 1988).

[11] S. Dietrich, M. Schick, Phys. Rev. B 33, 4952 (1985).

[12] I. Hadjiagapiou, R. Evans, Mol. Phys. 54, 383 (1985).

[13] E. Kierlik, M. L. Rosinberg, Y. Fan, P. Monson, J. Chem. Phys. 101, 10947 (1994).

[14] Y. Fan, J.E. Finn and P.A. Monson, J. Chem. Phys. 99, 8238 (1993).

[15] A. Plech, U. Klemradt, M. Huber, J. Peisl, Europhys. Lett., in press.

[16] See e.g. M.J.P. Nijmeijer and J.J. Weis in Annual Reviews of Computational Physics IV, World Scientific, Singapore (1996), and references therein.

[17] E.H. Hauge, Phys. Rev. B 33, 3322 (1986).

[18] J. M. J. Leeuwen, E. H. Hauge, J. Stat. Phys. 87, 1335 (1997); F. Clarysse, J. O. Indekeu, Physica A 251, 70 (1998).

[19] D. M. Kroll, G. Gompper, Phys. Rev. B 36, 7078 (1987); G. Gompper, D. M. Kroll, Phys. Rev. B 38, 459 (1988).

[20] F. F. Haas, F. Schmid, K. Binder, Phys. Rev. B, in print.

[21] C. J. Walden, B. L. Gyorffy, A. O. Parry, Phys. Rev. B 42, 798 (1990).

[22] S. Dietrich, M. Schick, Surf. Sci. 382, 178 (1997).

[23] Quantitative difference, e.g., in the prefactors, are due to the fact that the underlying potentials $f(m, \phi)$ differ from each other.

[24] J. Israelachvili. Intermolecular and surface forces (Academic Press, London, 1992).

[25] D. Frenkel and B. Smit, Understanding Molecular Simulation Academic Press, London (1996).

[26] D. Nicolaides and R. Evans, Phys. Rev. Lett. 63, 778 (1989).

[27] J.E. Finn and P.A. Monson, Phys. Rev. A39, 6402 (1989).

[28] Y. Fan and P.A. Monson, J. Chem. Phys. 99, 6897 (1993).

[29] H. Kellay, D. Bonn, J. Meunier, Phys. Rev. Lett. 71 2607, (1993).

[30] R. Pandit, M. E. Fisher, Phys. Rev. Lett. 51, 1772 (1983); J. Krim, J. G. Dash, J. Suzanne, Phys. Rev. Lett. 52, 640 (1984). 